\title{
Level of the SARS-CoV-2 receptor ACE2 is highly elevated in old-aged patients with aortic stenosis: implications for ACE2 as a biomarker for the severity of COVID-19
}

\section{Miklós Fagyas}

Division of Clinical Physiology, and Division of Cardiology, Department of Cardiology, Faculty of Medicine, University of Debrecen, Debrecen, Hungary

Attila Kertész

Department of Cardiology, Faculty of Medicine, University of Debrecen, Debrecen, Hungary Ivetta Siket Mányiné

Division of Clinical Physiology, Department of Cardiology, Faculty of Medicine, University of Debrecen, Debrecen, Hungary

\section{Viktor Bánhegyi}

Division of Clinical Physiology, Department of Cardiology and Doctoral School of Kálmán Laki, Faculty of Medicine, University of Debrecen, Debrecen, Hungary

\section{Bertalan Kracskó}

Division of Cardiology, Department of Cardiology and Doctoral School of Kálmán Laki, Faculty of Medicine, University of Debrecen, Debrecen, Hungary

\section{Andrea Szegedi}

Division of Cardiology, Department of Cardiology and Doctoral School of Kálmán Laki, Faculty of Medicine, University of Debrecen, Debrecen, Hungary

\section{Miklós Szokol}

Department of Cardiology, Faculty of Medicine, University of Debrecen, Debrecen, Hungary

\section{Gusztáv Vajda}

Division of Cardiology, Department of Cardiology, Faculty of Medicine, University of Debrecen, Debrecen, Hungary

\section{Ildikó Rácz}

Division of Cardiology, Department of Cardiology, Faculty of Medicine, University of Debrecen, Debrecen, Hungary

\section{Zoltán Csanádi}

Division of Cardiology, Department of Cardiology, Faculty of Medicine, University of Debrecen, Debrecen, Hungary

\section{Zoltán Papp}


Division of Clinical Physiology, Department of Cardiology, Faculty of Medicine, University of Debrecen, Debrecen, Hungary

\section{Attila Tóth ( $\nabla$ atitoth@med.unideb.hu )}

Division of Clinical Physiology, Department of Cardiology, Faculty of Medicine, University of Debrecen, Debrecen, Hungary https://orcid.org/0000-0001-6503-3653

\section{Sándor Sipka}

Division of Cardiology, Department of Cardiology, Faculty of Medicine, University of Debrecen, Debrecen, Hungary

\section{Research Article}

Keywords: ACE2, COVID-19, SARS-CoV-2, aortic stenosis, age

Posted Date: June 15th, 2020

DOI: https://doi.org/10.21203/rs.3.rs-34549/v1

License: (c) (1) This work is licensed under a Creative Commons Attribution 4.0 International License. Read Full License 


\section{Abstract}

Coronavirus disease 2019 (COVID-19) has a high mortality in elderly patients with pre- existing cardiovascular diseases. The cellular receptor of severe acute respiratory syndrome coronavirus 2 (SARSCoV-2) is the angiotensin converting enzyme 2 (ACE2), thereby implicating a link between cardiovascular diseases and SARS-CoV-2 susceptibility. Aortic stenosis (AS) represents a chronic inflammatory state with severe cardiovascular complications in the elderly, a prime condition for COVID-19 mortality. The circulating ACE2 levels were measured in 111 patients with severe AS and compared to patients with hypertension and healthy individuals.

About 4-times higher circulating ACE2 activity was found in patients with severe AS than in hypertensives or healthy individuals $(103.4 \pm 5.4, n=111,24.4 \pm 0.6, n=540$ and $17.3 \pm 1.3 \pm 0.6 \mathrm{mU} / \mathrm{L}, \mathrm{n}=46$, respectively). Patients with severe AS were older than patients with hypertension ( $79 \pm 0.7$ years vs. $60 \pm 0.5$ years, $P<0.05)$. Serum ACE2 activity correlated negatively with the left ventricular ejection fraction and positively with the right ventricular systolic pressure in patients with AS. In contrast, circulating ACE2 activity was independent of the blood pressure, characteristics of the stenotic aortic valve (aortic valve area, peak flow velocity), kidney function (GFR) and inflammatory state (CRP). We found no effect of RAAS inhibitory drugs on the serum ACE2 activity in this group of patients.

Our results illustrate circulating ACE2 as a potential interface between chronic inflammation, cardiovascular disease and COVID-19 susceptibility. Elderly patients with AS have markedly elevated ACE2 levels together with altered left and right ventricular functions, which may pose higher risks during COVID-19. Our clinical data do not support a role for RAAS inhibitors in regulating circulating ACE2 levels.

\section{Introduction}

Severe acute respiratory syndrome coronaviruses (SARS-CoV-1 and SARS-CoV-2) requires a cell surface receptor to bind to the target cells and to infect them. This receptor is the angiotensin converting enzyme 2 (ACE2) protein (Chen et al. 2020; Hoffmann et al. 2020; Tai et al. 2020; Walls et al. 2020). It was suggested that the binding of the spike protein of the SARS-CoV-2 to the cell surface located ACE2 is essential in viral infections (Lan et al. 2020). Accordingly, elevated circulating ACE2 levels were hypothetically linked to the severity of the disease (Walls et al. 2020; Zheng et al. 2020).

ACE2 is an important member of the Renin-Angiotensin-Aldosterone system (RAAS). It is believed to play a beneficial role by eliminating the ACE1 produced angiotensin II (Vaduganathan et al. 2020; Walls et al. 2020; Zheng et al. 2020). Our earlier studies implicated an association between circulating ACE2 levels and the progression of cardiovascular disease. In particular, circulating ACE2 levels were higher in hypertensive individuals (Fagyas et al. 2014) and in heart failure patients than in healthy individuals (Uri et al. 2016). ACE2 levels showed an apparent association with the left ventricular ejection fraction, and therefore, we proposed that ACE2 may play a role in the pathomechanism of cardiovascular disease development and progression (Uri et al. 2016). 
The coronavirus disease 2019 (COVID-19) has a devastatingly high mortality rate in the elderly (Grasselli et al. 2020). It was proposed that in countries with high mortality cardiovascular conditions may contribute to the vulnerability of patients. Most notably, hypertension has been recognized as a frequent comorbidity among SARS-CoV-2 contracted patients with severe complications (Grasselli et al. 2020). Interestingly, ageing of the immune system may also play an important role in influencing COVID-19 susceptibility. In this context, AS may identify a patient population with potentially severe cardiovascular complications as these patients are also known to suffer from a chronic inflammatory disorder (Cote et al. 2013).

We hypothesized that patients with severe AS have a dysregulated ACE-2. If this is true, then ACE2 may contribute to their vulnerability by providing a cellular receptor for SARS-CoV-2 infections.

\section{Methods}

\section{Ethical approval}

All studies were approved by the Regional and Institutional Ethics Committee, University of Debrecen, (UDCC REC/IEC number: 4375-2015) and by the Medical Research Council of Hungary. The research was in accordance with the tenets of the Helsinki Declaration.

\section{Patients}

Symptomatic patients with severe aortic valve stenosis awaiting transcatheter aortic valve implantation (TAVI) were enrolled. Data from our earlier studies were used for the healthy individuals and hypertensive patients (Uri et al. 2016; Uri et al. 2014). Patient's cardiovascular status was assessed by a detailed echocardiographic examination. The general patient's characteristics is summarized in Table 1.

\section{Samples}

Blood samples were collected according to the general aseptic technique in Vacutainer tubes (Cat. No. 367955, Becton Dickinson, Franklin Lakes, NJ, USA). Blood samples were left at room temperature to clot for about $30 \mathrm{~min}$. After clotting completed, serum (supernatant) was collected by centrifuging at 1,500 $\mathrm{g}$ at room temperature for $15 \mathrm{~min}$. Samples were then frozen $\left(-20^{\circ} \mathrm{C}\right)$ until the biochemical measurements.

Blood samples were obtained before transcatheter aortic valve implantation (TAVI), when a detailed echocardiographic assessment was performed.

\section{Measurement of the serum ACE2 activity}

Serum ACE2 activity was measured as described before (Fagyas et al. 2014; Uri et al. 2016) with some modifications. In short, ACE2 activity was measured from $20 \mathrm{ml}$ of sera in a black 96 -well microtiter plate (Greiner Bio-One, Frickenhauser, Germany). It was supplemented by $80 \mathrm{ml}$ of assay buffer ( $75 \mathrm{mM}$ Tris$\mathrm{HCl}, \mathrm{pH}$ 6.5). Plate was warmed to $37^{\circ} \mathrm{C}$. Then $100 \mathrm{ml}$ of substrate-salt solution (containing $1.333 \mathrm{M}$ 
$\mathrm{NaCl}, 26.7 \mathrm{mM} Z \mathrm{ZnCl}$, $20 \mathrm{mM}$ bestatin-hydrochlorid, $20 \mathrm{mM}$ Z-prolyl-prolinal, $10 \mathrm{mM}$ amastatinhydrochloride, 20 mM captopril, 6 mM PMSF and 100 mM Mca-APK(Dnp) (((7-methoxycoumarin-4yl)acetyl-Ala-Pro-Lys(2,4-dinitrophenyl)-OH), from EZ Biolab, Parsippany, NJ, USA) in assay buffer) was added to start the reaction. The reaction was run at $37^{\circ} \mathrm{C}$. The cleavage of the quenched Mca-APK(Dnp) to liberate the fluorescent $\mathrm{K}(\mathrm{Dnp})$ were recorded using $340 \mathrm{~nm}$ excitation and $405 \mathrm{~nm}$ emission filters in a Novostar (BMG Labtech, Ortenberg, Germany) microplate reader. The fluorescent intensities (in arbitrary units, $A U$ ) were plotted as the function of the reaction time. The slope of the linear fit on the plotted data yielded the measure of the activity (in AU/min) and accepted only if the goodness of the fit $\left(\mathrm{r}^{2}\right)$ was higher than 0.9. The actual amount $(\mathrm{pmol})$ of the converted substrate was calculated based on the calibration of the instrument with the known concentration (amount) of the fully cleaved substrate. The unit (U) of activity is defined as micromole/min.

\section{Statistical analysis}

Mann-Whitney test was used when two groups were compared. Kruskal-Wallis test with Dunn's multiple comparisons test was performed for multiple groups by Prism6 for Mac OS X (GraphPad Software, San Diego, CA, USA). Differences were considered to be significant when the statistical software indicated $(P<0.05$ in Mann-Whitney test or ranks being significant, alpha was set to 0.05 in the Kruskal-Wallis test).

\section{Results}

\section{Aortic stenosis associates with high ACE2 activities in the elderly}

Mean ACE2 activity in patients with severe aortic stenosis (AS) $(103.4 \pm 5.4 \mathrm{mU} / \mathrm{L}, \mathrm{n}=111$ vs. $24.4 \pm 0.6$ $\mathrm{mU} / \mathrm{L}, \mathrm{n}=540$, Fig. $1 \mathrm{~A}$ and $1 \mathrm{~B}$ ) was about 4-fold higher than in hypertensive patients and 6-fold higher than in healthy individuals $(17.3 \pm 1.3 \mathrm{mU} / \mathrm{L}, \mathrm{n}=46,1 \mathrm{~A}$ and $1 \mathrm{~B})$. Patients with $\mathrm{AS}$ represented an older population than the healthy group or hypertensive individuals ( $79 \pm 0.7$ years, $n=111,60 \pm 0.5$ years and $38 \pm 1.3$ years, respectively, Fig. 1A and 1C).

\section{Inhibition of the RAAS system does not modulate ACE2 activity in AS}

We did not find a statistically significant difference in serum ACE2 activities in patients with AS treated without RAASi (No RAASi, 108 $\pm 19 \mathrm{mU} / \mathrm{L}, \mathrm{n}=16$, Fig. 2), treated with ACE inhibitors (ACEi, $107 \pm 7 \mathrm{mU} / \mathrm{L}$, $n=68$, Fig. 2), with angiotensin receptor blockers (ARB, $89 \pm 10 \mathrm{mU} / \mathrm{L}, \mathrm{n}=15$, Fig. 2) or with aldosterone antagonists (AA, $102 \pm 19 \mathrm{mU} / \mathrm{L}, \mathrm{n}=11$, Fig. 2).

\section{Circulating ACE2 activity correlates with some, but not all hemodynamic indices of AS}

Efforts were made to identify potential correlations of elevated serum ACE2 activities with cardiovascular parameters of AS patients. Serum ACE2 activity was highly elevated (360\% increase) in severe AS patients with maintained left ventricular ejection fraction ( $E F>50 \%, 88 \pm 6 \mathrm{mU} / \mathrm{L}, \mathrm{n}=57$, Fig. $3 \mathrm{~A}$ ), but not in patients with hypertension (having similar EF values). Left ventricular systolic dysfunction (EF $\leq 50 \%)$ did 
not result in a significant difference in serum ACE2 activities (nominal increase to $121 \pm 8 \mathrm{mU} / \mathrm{L}, \mathrm{n}=53$, Fig. $3 A$ ) in severe AS patients. The correlation of serum ACE2 activity was then studied with various clinical parameters. Correlations were considered to exist when the line reasonably fitted the measured values $\left(r^{2}\right.$ values reached the arbitrary threshold of $0.1(r>0.3))$ and when the fitted line significantly deviated $(P<0.05)$ from a horizontal line. Serum ACE2 activity was independent of blood pressure (either systolic, Fig. 3B or diastolic, Fig. 3C). Similar results (no correlation) was found with the thickness of septum in the heart (Fig. 3D). In contrast, serum ACE2 activity positively correlated with the diameter of the endsystolic left ventricular diameter (systolic diameter, Fig. 3E) and a borderline correlation was seen with the left ventricular diastolic diameter, Fig. 3F). Nonetheless, serum ACE2 activity negatively correlated with the overall measure of left ventricular systolic function (EF, Fig. 3G).

Serum ACE2 activity did not correlate with kidney function (GFR, Fig. 4A) and acute inflammatory states (CRP, Fig. 4B). The severity of the aortic stenosis (aortic valve cross-sectional area, Fig. 4C and maximal blood flow velocity, Fig. 4D) was also independent of the increase in serum ACE2 activity in AS patients.

A borderline correlation of serum ACE2 activity was seen with the left atrial diameter (positive correlation, Fig. 5A) and tricuspid annular plane systolic excursion (TAPSE, negative correlation, Fig. 5B). In accordance, the calculated right ventricular systolic pressure showed a positive correlation with the serum ACE2 activity (Fig 5C). Finally, a composite measure representing both left and right ventricular alterations was calculated (right ventricular systolic pressure / left ventricular ejection fraction). This parameter showed the strongest correlation with the serum ACE2 activity (Fig. 5D).

\section{Discussion}

Elderly patients with cardiovascular complications are the most susceptible for COVID-19 mortality (Zheng et al. 2020). The explanation for the apparent age-dependence still remains as a mystery, although comorbidities are frequently cited in the elderly. In this context, the kind and severity of cardiovascular disease has been extensively discussed. Most recently it was also suggested at the highest levels of professional media, that the cellular receptor for SARS-CoV-2, ACE2 can link cardiovascular disease to SARS-CoV-2 susceptibility (Esler and Esler 2020; Vaduganathan et al. 2020; Zheng et al. 2020).

We assumed that patients with severe aortic stenosis (AS) may represent a prime population of patients to study ACE2 levels in relation to COVID-19 mortality. AS is characterized by a chronic inflammatory process (Cho et al. 2018; Cote et al. 2013), leading to narrowing of the aortic root, elevated left ventricular pressure levels, and a passive backward transmission of filling pressures that increases pulmonary (and right ventricular) blood pressure (Sysol and Machado 2018), all of these being implicated in COVID-19 mortality (Zheng et al. 2020). It has also been proposed that cardiovascular medication, in particular, inhibitors of the Renin-Angiotensin-Aldosterone system (RAASi) elevate serum ACE2 levels and potentially contribute to the mortality of COVID-19 (Vaduganathan et al. 2020; Walls et al. 2020; Zheng et al. 2020). 
The prevalence of aortic stenosis (AS) is estimated to be $12.4 \%$ in the general elderly population ( $\geq 75$ years of age) in Europe and US. The severe form of this disease, which was targeted in this study, is about 3.4\% (Osnabrugge et al. 2013). In accordance with these figures, our severe AS patient population had a mean age of 79 years.

We compared the AS population with a hypertensive population, to establish the role of ageing and additional factors over elevated left ventricular systolic pressure. Indeed, the serum ACE2 activity was 4fold higher in the patients with severe AS than that in hypertensive patients, suggesting that hypertension itself is probably not the primary determinant of these elevated ACE2 levels. As a matter of fact, the circulating ACE2 activity did not correlate with systolic or diastolic blood pressure, or left ventricular wall thickness in the severe AS patients, suggesting that ACE2 dysregulation is not the result of hypertension, per se. Then we estimated the contribution of the left ventricular systolic dysfunction, which had an effect on circulating ACE2 levels in heart failure patients with reduced ejection fraction (HFrEF) (Fagyas et al. 2014; Uri et al. 2016). A weak correlation was established, confirming earlier results, but it was not likely to be a major determinant in the severe AS patients for two reasons. First, the severe AS patients with preserved left ventricular ejection fraction $(E F \geq 50 \%)$ had similar serum ACE2 activities as severe AS patients with reduced $E F(E F<50 \%)$. Second, there was an almost four-fold difference in serum ACE2 activities between hypertensive patients with maintained $E F(E F>50 \%)$ and severe AS patients with similarly maintained EF. These suggested that factor(s) other than systolic dysfunction may contribute to ACE2 dysregulation in severe AS patients.

Severe AS often result in a pulmonary congestion, due to retrograde transmission of elevated filling pressures in the left heart, leading directly to an increase in pulmonary artery blood pressure (Sysol and Machado 2018). Almost all patients in our severe AS population had an elevated $(>25 \mathrm{mmHg}$ ) right ventricular pressure, defining these patients as group 2 pulmonary hypertensives (Sysol and Machado 2018). We identified an association between serum ACE2 activity and the calculated right ventricular systolic blood pressure (based on echocardiography). The state and alterations of in COVID-19 infected patients is not clear yet, but probably include a dysregulation of the pulmonary circulation (Gattinoni et al. 2020), which may be paralleled by dysregulation of the ACE2 levels.

Finally, it gained a high public profile when several groups extrapolated the preclinical data suggesting that treatment of cardiovascular patients with RAAS inhibitors (primarily chosen drugs in hypertension and heart failure) may result in an increase in serum ACE2 levels (Fang et al. 2020; Vaduganathan et al. 2020; Zheng et al. 2020). Our data did not support this extrapolation from animal experiments. We did not find a difference in circulating ACE2 levels in patients treated with ACEi, with ARB or without RAAS inhibitors, suggesting that the role for medication is insignificant in determining circulating ACE2 levels in severe AS patients.

Taken together, here we showed, that elderly patients with severe aortic stenosis have a four-fold higher circulating ACE2 levels, than hypertensive patients. This elevation in ACE2 levels can be the combined effect of reduced left ventricular systolic function, elevated pulmonary pressure and age in this patient 
population. Among these factors, pulmonary congestion and age seems to be dominant. Our data suggest that circulating ACE2 may reflect susceptibility for SARS-CoV-2 infections. Moreover, we propose that circulating ACE2 activity can be considered as a cardiovascular biomarker with potential implications for SARS-CoV-2 infections.

\section{Declarations}

\section{Ethical approval}

All studies were approved by the Regional and Institutional Ethics Committee, University of Debrecen, (UDCC REC/IEC number: 4375-2015) and by the Medical Research Council of Hungary. The research was in accordance with the tenets of the Declaration of Helsinki. All individuals involved in the studies gave their informed, written consent before enrolment The Authors declare no conflict of interest.

\section{Financial Sources}

The research was financed by the Thematic Excellence Programme of the Ministry for Innovation and Technology in Hungary (ED_18-1-2019-0028), within the framework of the thematic programme of the University of Debrecen (to AT). The work is supported by the GINOP-2.3.2-15-2016-00043 (to ZCs, IÉ, ZP and AT), GINOP-2.3.2-15-2016-00050 (to AT) and EFOP-3.6.2-16-2017-00006 (to ZP and AT) projects. These projects are co-financed by the European Union and the European Regional Development Fund (GINOP) and the European Social Fund (EFOP). Projects FK 128809 (to MF), K 116940 and K 132623 (to AT) have been implemented with the support provided from the National Research, Development and Innovation Fund of Hungary.

\section{Tables}

Table 1. Clinical characteristics of the patients 


\begin{tabular}{ll} 
General characteristics & Values \\
\hline Age (years) & $79 \pm 0.7$ \\
\hline Male sex (\%) & 38.7 \\
\hline Hypertension (\%) & 83.8 \\
\hline Diabetes Mellitus (\%) & 40.2 \\
\hline Hyperlipidemia (\%) & 45 \\
\hline Peripheral vascular disease (\%) & 5.4 \\
\hline Coronary Artery disease (\%) & 41.8 \\
\hline Cardiac decompensation in history (\%) & 49.5 \\
\hline COPD (\%) & 12.6 \\
\hline Atrial Fibrillation (\%) & 46.8
\end{tabular}

Pharmacotherapy of the patients

\begin{tabular}{ll} 
Type of medication & $\%$ of patients \\
\hline Acetylsalicylic acid (ASA) & 45.9 \\
\hline Clopidogrel & 69.4 \\
\hline Vitamin K antagonist & 22.5 \\
\hline Direct Oral Anticoagulant & 20.7 \\
\hline ACE Inhibitor & 62.2 \\
\hline ARB & 12.6 \\
\hline Beta Blocker & 79.2 \\
\hline Statin & 57.6 \\
\hline Mineralocorticoid receptor antagonist (MRA) & 38.7 \\
\hline Furosemide & 79.3
\end{tabular}

\section{Echocardiographic parameters}


Echocardiographic parameters

Ejection fraction (EF, \%)

$49.1 \pm 9.5$

Septal thickness (mm)

$13.61 \pm 1.8$

Posterior wall thickness $(\mathrm{mm})$

$13.42 \pm 1.4$

Left ventricle end-systolic diameter $(\mathrm{mm})$

$32.13 \pm 7.7$

Left ventricle end-diastolic diameter $(\mathrm{mm})$

$52.61 \pm 6.3$

Left atrial diameter $(\mathrm{mm})$

$44.73 \pm 5.4$

Mitral regurgitation grade (MR)

$1.42 \pm 0.6$

Patients with moderate and severe regurgitation (MR, AR, TR) $(\geq 2, \%)$

$29.73(33 / 111)$

Aortic valve area $\left(\mathrm{cm}^{2}\right)$

$0.59 \pm 0.14$

Aortic peak gradient $(\mathrm{mmHg})$

$74.79+20.2$

Aortic mean gradient $(\mathrm{mmHg})$

$47.32+13.2$

Aortic regurgitation grade (AR)

$1.44 \pm 0.7$

Patients with moderate and severe $\operatorname{AR}(\geq 2, \%)$

$29.73(33 / 111)$

Tricuspid regurgitation grade (TR)

$1.45 \pm 0.6$

Patients with moderate and severe TR $(\geq 2, \%)$

$31.5(35 / 111)$

Pulmonary arterial pressure $(\mathrm{mmHg})$

$47.81 \pm 10.9$

TAPSE $(\mathrm{mm})$

$19.43 \pm 3.6$

\section{References}

Chen Y, Guo Y, Pan Y, Zhao ZJ (2020) Structure analysis of the receptor binding of 2019-nCoV Biochemical and biophysical research communications doi:10.1016/j.bbrc.2020.02.071

Cho KI, Sakuma I, Sohn IS, Jo SH, Koh KK (2018) Inflammatory and metabolic mechanisms underlying the calcific aortic valve disease Atherosclerosis 277:60-65 doi:10.1016/j.atherosclerosis.2018.08.029

Cote $\mathrm{N}$ et al. (2013) Inflammation is associated with the remodeling of calcific aortic valve disease Inflammation 36:573-581 doi:10.1007/s10753-012-9579-6

Esler M, Esler D (2020) Can angiotensin receptor-blocking drugs perhaps be harmful in the COVID-19 pandemic? Journal of Hypertension Publish Ahead of Print doi:10.1097/hjh.0000000000002450

Fagyas $\mathrm{M}$ et al. (2014) New perspectives in the renin-angiotensin-aldosterone system (RAAS) II: albumin suppresses angiotensin converting enzyme (ACE) activity in human PloS one 9:e87844 
doi:10.1371/journal.pone.0087844

Fang L, Karakiulakis G, Roth M (2020) Are patients with hypertension and diabetes mellitus at increased risk for COVID-19 infection? Lancet Respir Med doi:10.1016/s2213-2600(20)30116-8

Gattinoni L, Coppola S, Cressoni M, Busana M, Rossi S, Chiumello D (2020) Covid-19 Does Not Lead to a "Typical" Acute Respiratory Distress Syndrome American journal of respiratory and critical care medicine doi:10.1164/rccm.202003-0817LE

Grasselli G et al. (2020) Baseline Characteristics and Outcomes of 1591 Patients Infected With SARSCoV-2 Admitted to ICUs of the Lombardy Region, Italy Jama doi:10.1001/jama.2020.5394

Hoffmann M et al. (2020) SARS-CoV-2 Cell Entry Depends on ACE2 and TMPRSS2 and Is Blocked by a Clinically Proven Protease Inhibitor Cell doi:10.1016/j.cell.2020.02.052

Lan J et al. (2020) Structure of the SARS-CoV-2 spike receptor-binding domain bound to the ACE2 receptor Nature doi:10.1038/s41586-020-2180-5

Osnabrugge RL et al. (2013) Aortic stenosis in the elderly: disease prevalence and number of candidates for transcatheter aortic valve replacement: a meta-analysis and modeling study Journal of the American College of Cardiology 62:1002-1012 doi:10.1016/j.jacc.2013.05.015

Sysol JR, Machado RF (2018) Classification and pathophysiology of pulmonary hypertension Continuing Cardiology Education 4:2-12 doi:10.1002/cce2.71

Tai W et al. (2020) Characterization of the receptor-binding domain (RBD) of 2019 novel coronavirus: implication for development of RBD protein as a viral attachment inhibitor and vaccine Cellular \& Molecular Immunology doi:10.1038/s41423-020-0400-4

Uri K et al. (2016) Circulating ACE2 activity correlates with cardiovascular disease development J Renin Angiotensin Aldosterone Syst 17 doi:10.1177/1470320316668435

Uri $\mathrm{K}$ et al. (2014) New perspectives in the renin-angiotensin-aldosterone system (RAAS) IV: circulating ACE2 as a biomarker of systolic dysfunction in human hypertension and heart failure PloS one 9:e87845 doi:10.1371/journal.pone.0087845

Vaduganathan M, Vardeny O, Michel T, McMurray JJV, Pfeffer MA, Solomon SD (2020) ReninAngiotensin-Aldosterone System Inhibitors in Patients with Covid-19 The New England journal of medicine doi:10.1056/NEJMsr2005760

Walls AC, Park YJ, Tortorici MA, Wall A, McGuire AT, Veesler D (2020) Structure, Function, and Antigenicity of the SARS-CoV-2 Spike Glycoprotein Cell doi:10.1016/j.cell.2020.02.058 
Zheng YY, Ma YT, Zhang JY, Xie X (2020) COVID-19 and the cardiovascular system Nat Rev Cardiol doi:10.1038/s41569-020-0360-5

\section{Figures}
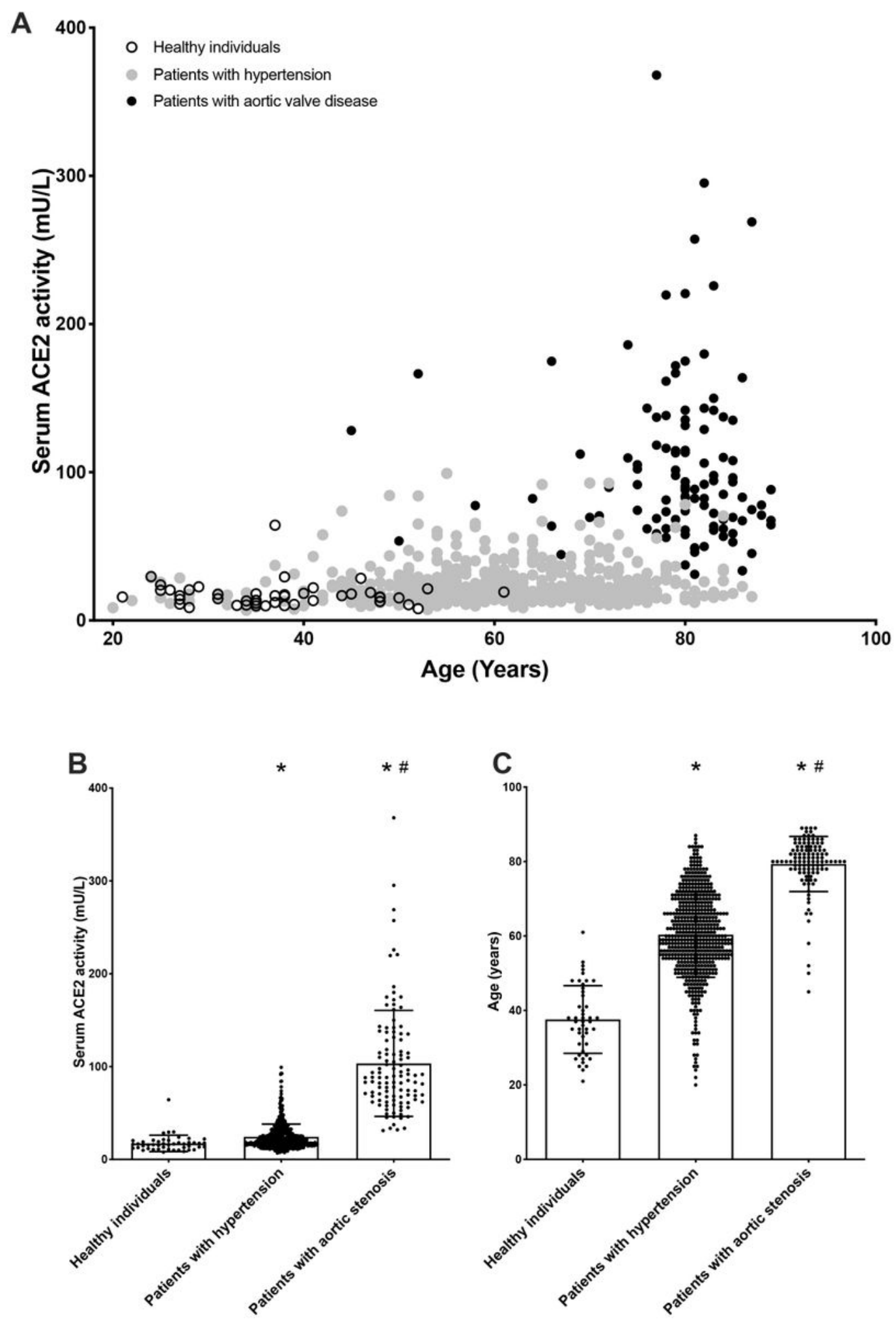

Figure 1 
Elderly patients with severe aortic stenosis have increased ACE2 activity Patients with hypertension $(n=540)$, severe aortic stenosis $(n=111)$ and healthy individuals $(n=46)$ were enrolled in this evaluation. Panel A: Levels of serum ACE2 activity are plotted as a function of the age. Each symbol represents an individual (open circles: healthy individuals, grey filled circles: patients with hypertension, black filled circles: patients with severe aortic valve disease). Panel B: Comparison of the serum ACE2 activities of the patient's groups. Panel C: Comparison of the age of the patient's groups. Columns are mean, bars are SEM, symbols represent the individual values. Significant differences are indicated by the asterisks (from the healthy individuals) or by the hash (from the hypertensive group).

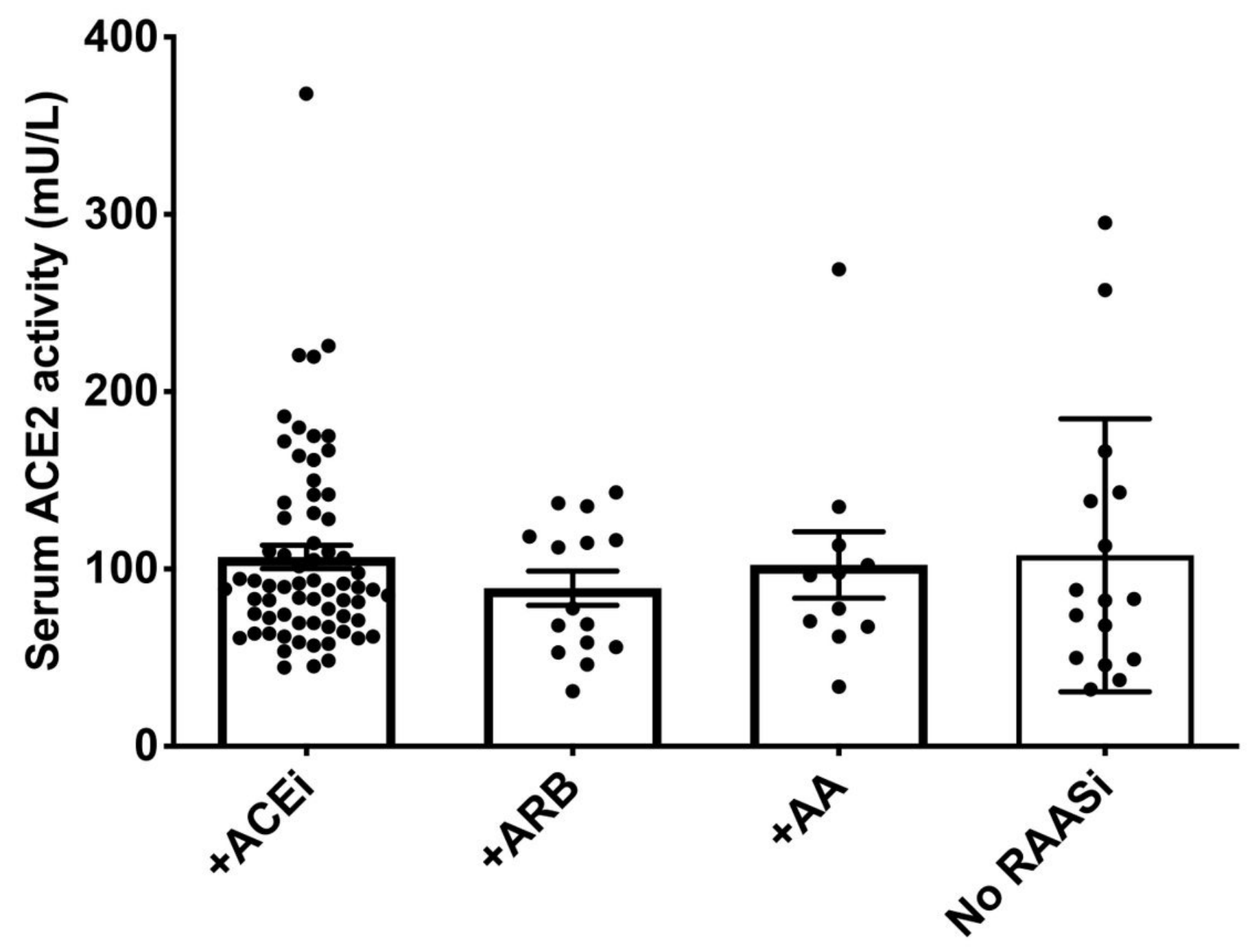

\section{AS patients treated with}

Figure 2

RAAS inhibitory medication is without effects on the serum ACE2 activities Serum ACE2 activity of patients with severe aortic stenosis is plotted on the graph. Patients with prescriptions for angiotensin 
converting enzyme inhibitors (+ACEi, $n=68$ ), with angiotensin receptor blockers (+ABR, $n=15$ ), with aldosterone antagonists (+AA, $n=11)$ and without prescribed Renin-Angiotensin-Aldosterone system inhibitors (No RAASi, $n=16$ ) are represented. Symbols represent individual values; columns are the mean and bars are SEM. None of the groups differ significantly.

B

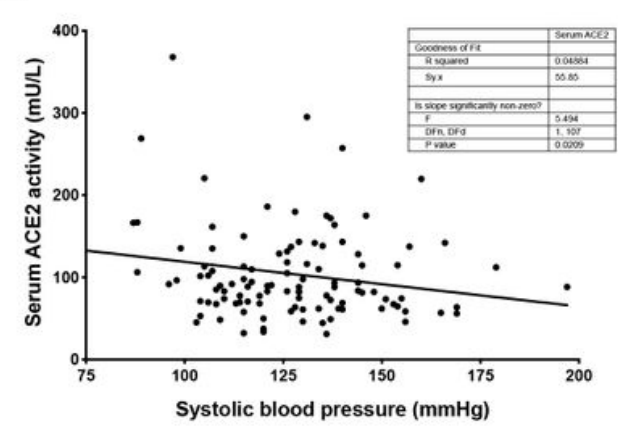

E

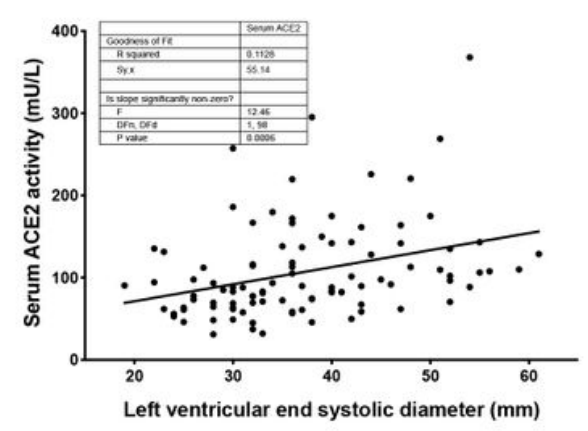

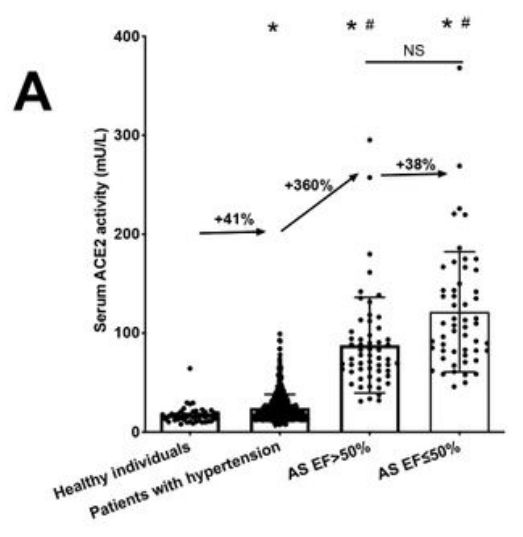

C

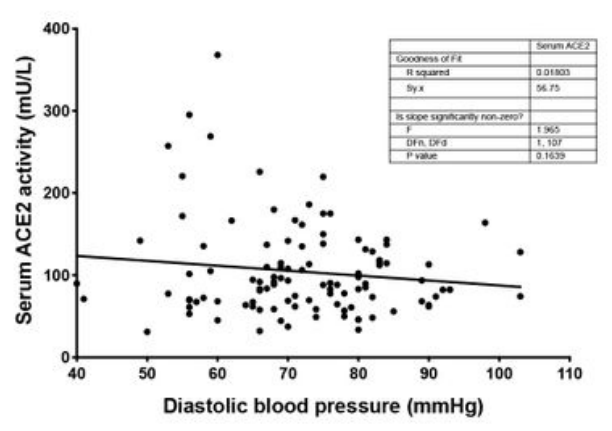

$\mathbf{F}$

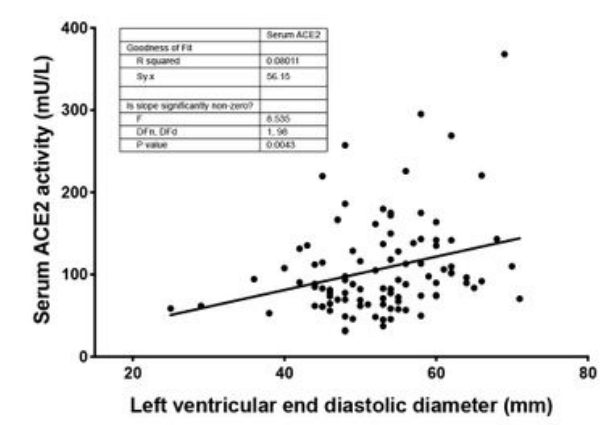

D

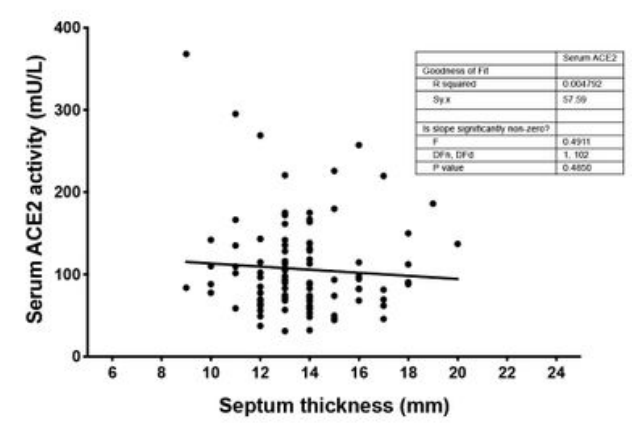

G

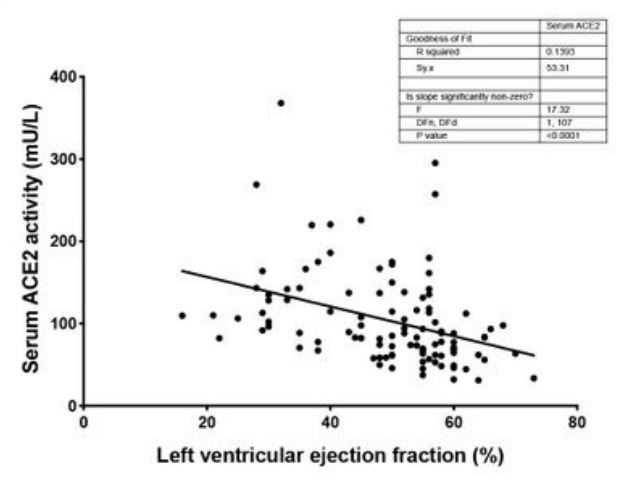

Figure 3

Serum ACE2 activity are increased in AS patients: a correlation with systolic function Panel A: Serum ACE2 activities of the different clinical groups (healthy, $n=46$; patients with hypertension, $n=540$; patients with severe aortic stenosis having maintained left ventricular systolic function (AS EF>50\%, $n=57$ ); patients with severe aortic stenosis having reduced left ventricular systolic function (AS EF $\leq 50 \%, n=53)$ ). Each symbol is an individual value, the arrows represent the mean increase in serum ACE2 values the values are \% of increase. Columns and bars show the mean and SEM. Significant differences are indicated by the asterisks (from the healthy individuals) or by the hashes (from the hypertensive group). Correlations of serum ACE2 activities were also tested with systolic blood pressure (Panel B), diastolic 
blood pressure (Panel C), thickness of the interventricular septum (Panel D), diameter of the left ventricle (end-systolic, Panel E, end-diastolic, Panel F), left ventricular ejection fraction (Panel G). Each symbol represents an individual patient; the line is the linear regression fitted on the values. The data tables show the parameters of the fits.
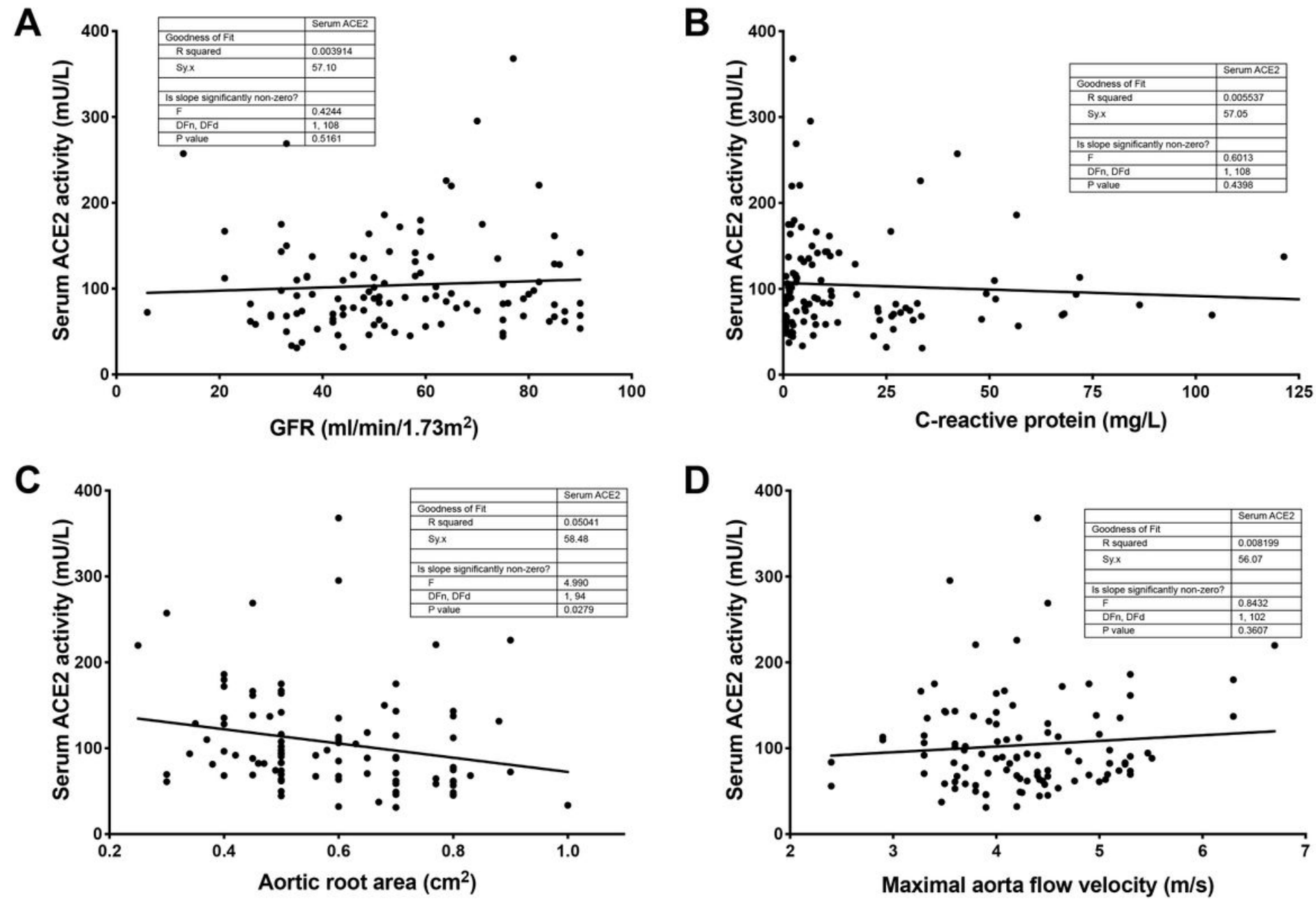

\section{Figure 4}

Serum ACE2 activity do not correlate with parameters of kidney function, inflammation or the severity of the aortic stenosis Serum ACE2 activities were plotted as the function of the glomerular filtration rate (GFR, Panel A), C-reactive protein (CRP, Panel B), aortic valve area (Panel C) and peak aortic flow velocity (Panel D). Each symbol represents an individual patient; the line is the linear regression fitted on the values. The data tables show the parameters of the fits. 
A

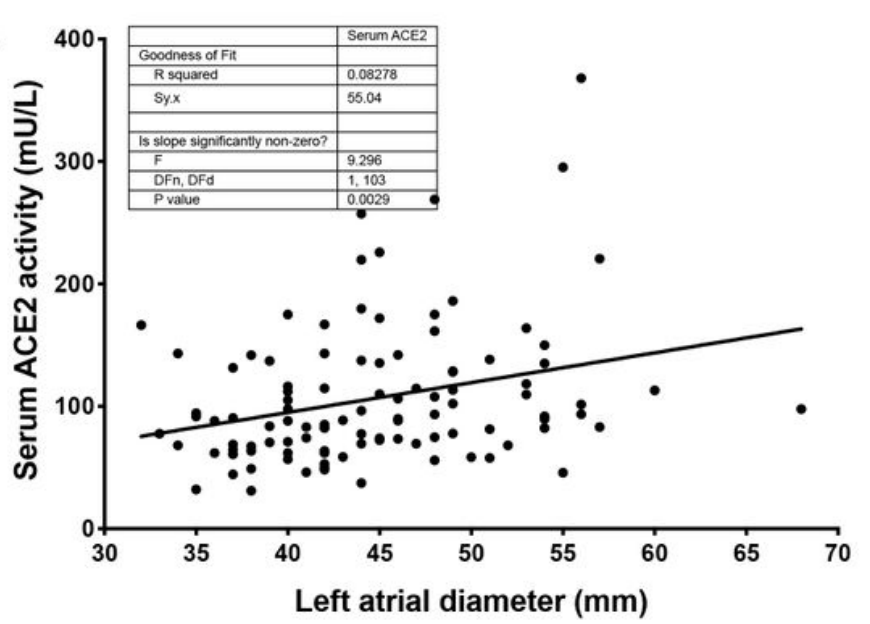

C

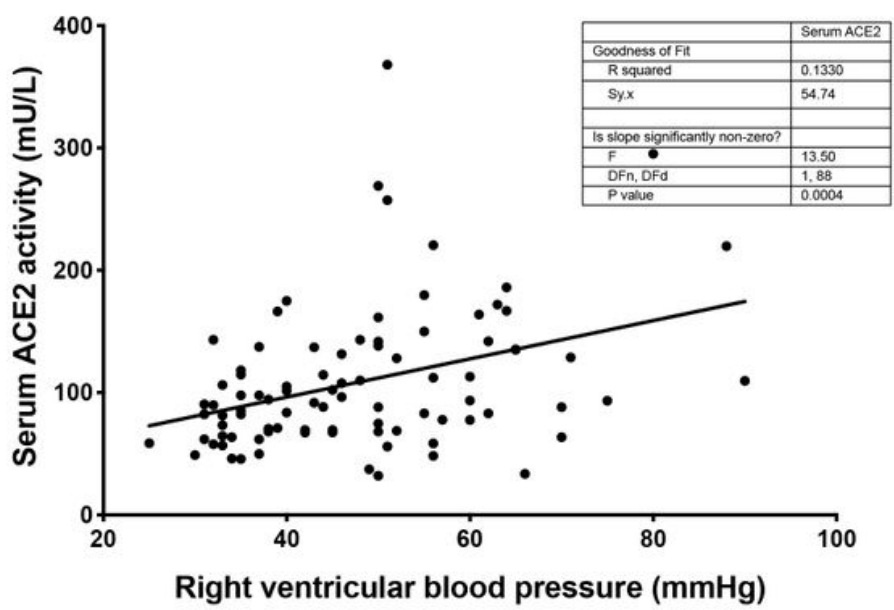

B

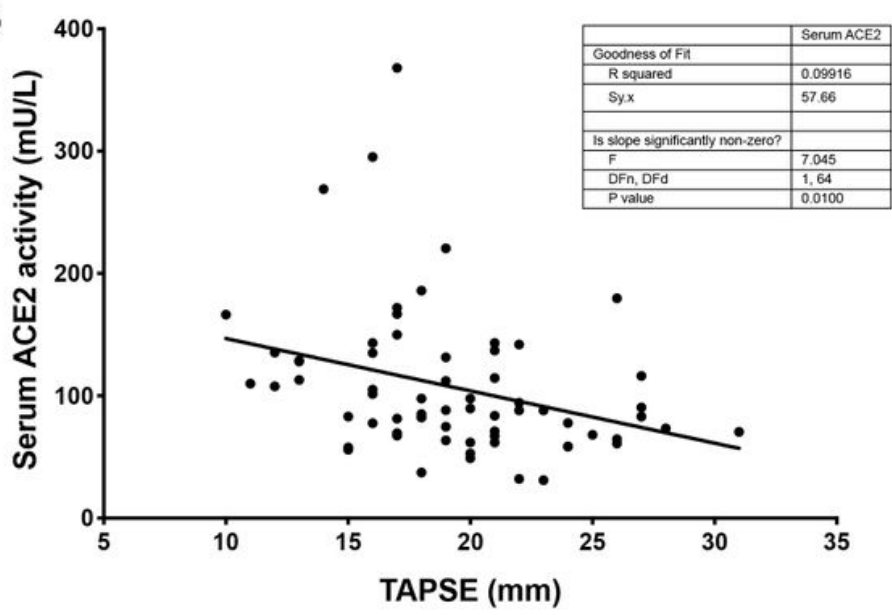

D

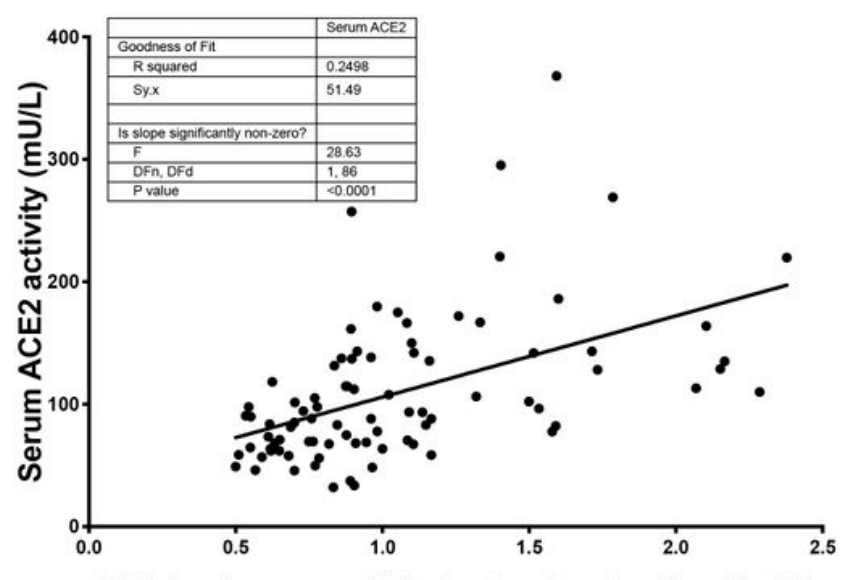

RV blood pressure/LV ejection fraction $(\mathrm{mmHg} / \%)$

\section{Figure 5}

Serum ACE2 levels correlate with right ventricular systolic pressure Serum ACE2 activities were plotted as the function of the left atrial diameter (Panel A), Tricuspid Annular Plane Systolic Excursion (TAPSE, Panel B), right ventricular systolic blood pressure (Panel C) and a composite product of the right ventricular systolic pressure (RV blood pressure) and the left ventricular ejection fraction (LV ejection fraction, Panel D). Each symbol represents an individual patient; the line is the linear regression fitted on the values. The data tables show the parameters of the fits. 